\title{
PERIODIC CYCLIC COHOMOLOGY CHERN CHARACTER FOR PSEUDOMANIFOLDS WITH ONE SINGULAR STRATUM
}

\author{
SHING-WAI CHAN \\ (Communicated by Leslie Saper)
}

\begin{abstract}
We compute the periodic cyclic cohomology Chern character of an admissible pseudomanifold $X^{\dagger}$ with one singular stratum. As a corollary, we obtain the index theorem and spectral flow for signature operators.
\end{abstract}

\section{INTRODUCTION}

This paper is the sequel to [Chan]. In that paper, by considering the "straight" Chern character, we gave a de-Rham type realization of the Goresky-MacPhersonSiegel $\mathcal{L}$-classes of admissible pseudomanifolds with one singular stratum $X^{\dagger}=$ $M \cup\left(c^{\dagger}(L) \times N\right)$ such that $2 L$ has zero oriented cobordism, and we also obtained the index theorem for the twisted signature operators on $X^{\dagger}$. In this paper, we will continue to study $X^{\dagger}$ by using non-commutative geometry.

We will compute (Theorem 3.1 and Theorem 3.3) the corresponding periodic cyclic cohomology Chern character by using the infinite temperature limit formula in $[\mathrm{CoM}]$. As in [Chan], we will choose a scaling in the conical direction such that the signature operator is essentially self-adjoint. Also, by using a singular elliptic estimate, we handle the calculation on the singular part by that on model space $c_{0, \infty}(L) \times N$. We finish the computation by Getzler's calculus. As a consequence, we recover the index theorem for twisted signature operators on $X^{\dagger}$ [Chan, Theorem 4.1 in the even case. In the odd case, we obtain (Corollary 3.4) the spectral flow for signature operators on admissible spaces with conical singularity.

\section{Preliminaries}

To fix the notation, let us recall some definitions in [Chan].

Let $M$ be a smooth, oriented, compact and connected $m$-dimensional manifold with boundary $\partial M=L \times N$ where $L$ and $N$ are smooth, oriented, closed and connected manifolds of dimensions $\ell$ and $n$ respectively. Let $c(L)=(0,1) \times L$ and $c^{\dagger}(L)=[0,1) \times L /\{0\} \times L$ be the cone and completed cone with link $L$ respectively.

Then $X^{\dagger}=M \cup\left(c^{\dagger}(L) \times N\right)$ is called a pseudomanifold with one singular stratum [Chan]. We define a metric $g$ on $X^{\dagger}$ such that

(i) $g$ is a measurable metric on $X^{\dagger}$;

(ii) $\left.g\right|_{M}$ is a smooth metric on $M$ and is a product near $\partial M$;

Received by the editors August 30, 1996.

1991 Mathematics Subject Classification. Primary 19D55; Secondary 58G12.

(C)1998 American Mathematical Society 
(iii) $\left.g\right|_{c^{\dagger}(L) \times N}=\left(d r^{2}+\psi(r)^{2} g^{L}\right) \oplus g^{N}$, where $g^{L}$ and $g^{N}$ are smooth metrics on $L$ and $N$ respectively, and $\psi:[0,1] \rightarrow[0,1]$ is a $C^{\infty}$ function such that

$$
\psi(r)= \begin{cases}r, & r \in\left[0, \frac{2}{3}\right] \\ 1, & r \in\left[\frac{3}{4}, 1\right]\end{cases}
$$

and $\psi(r) \neq 0$ for $r>0$.

Clearly, this is a Lipschitz metric.

$X^{\dagger}$ is called admissible if $\ell=\operatorname{dim} L$ is odd or $H^{\frac{\ell}{2}}(L)=0$.

The stratified form $\Omega^{*}\left(X^{\dagger}\right)_{S A}$ is defined as follows:

$\Omega^{*}\left(X^{\dagger}\right)_{S A}=$ the subspace of Lipschitz forms on $X^{\dagger}$ with restriction to $M$ belonging to $\Omega^{*}(M)$, and on $c(L) \times N=(0,1) \times L \times N$, it is the pull-back of a form from $\Omega^{*}(N)$.

$\Omega^{0}\left(X^{\dagger}\right)_{S A}$ is an algebra, which will also be denoted by $C_{S A}^{\infty}\left(X^{\dagger}\right)$.

\section{Infinite temperature limit FORMula FOR CHERN CHARACTER}

In this section, we will recall the infinite temperature limit formula for the Chern character in non-commutative geometry $[\mathrm{CoM}]$. We will follow the notation in $[\mathrm{CoM}]$ closely.

Let $(\mathcal{H}, D)$ be an unbounded $p$-summable Fredholm module over $\mathcal{A}$ and $\Delta_{n}=$ $\left\{\left(t_{1}, \ldots, t_{n}\right) \in \mathbb{R}^{n}: 0 \leq t_{1} \leq \cdots \leq t_{n} \leq 1\right\}$. Given $A_{0}, \ldots, A_{n} \in B(\mathcal{H})$, we set

$$
\left\langle A_{0}, \ldots, A_{n}\right\rangle_{D}=c \int_{\Delta_{n}} \operatorname{Tr}\left(\gamma A_{0} e^{-t_{1} D^{2}} A_{1} e^{-\left(t_{2}-t_{1}\right) D^{2}} \cdots A_{n} e^{-\left(1-t_{n}\right) D^{2}}\right) d t_{1} \cdots d t_{n},
$$

where $c=1$ if $(\mathcal{H}, D)$ is graded and $c=\sqrt{2 i}, \gamma=I$ if $(\mathcal{H}, D)$ is ungraded.

With this notation, the JLO-cocycle representing the entire Chern character of $(\mathcal{H}, D)$ is given by the following components:

$$
C h^{n}(D)\left(a^{0}, \ldots, a^{n}\right)=\left\langle a^{0},\left[D, a^{1}\right], \ldots,\left[D, a^{n}\right]\right\rangle_{D}, \quad a^{0}, \ldots, a^{n} \in \mathcal{A},
$$

where $n$ runs through all positive integers of the same parity as $(\mathcal{H}, D)$. Also, for an operator $V$ on $\mathcal{H}$ of degree $[V] \in \mathbb{Z}_{2}$, we define

$$
=\sum_{0 \leq i \leq n}(-1)^{i[V]}\left\langle a^{0},\left[D, a^{1}\right], \ldots,\left[D, a^{i}\right], V,\left[D, a^{i+1}\right], \ldots,\left[D, a^{n}\right]\right\rangle_{D}
$$

Suppose $\phi(t)$ is a vector-valued function that satisfies the following condition:

$(\sharp) \quad$ There exist $T>0$ such that on $(0, T]$, we have

$$
\phi(t)=\psi(t)+\sum_{1 \leq k \leq K}\left[\alpha_{k}+\sum_{1 \leq j \leq J} \alpha_{k j} \log ^{j} t\right] t^{-v_{k}}+\sum_{1 \leq k \leq K}\left[\beta_{k}+\sum_{1 \leq j \leq J} \beta_{k j} t \log ^{j} t\right] t^{-k}
$$

where $\psi(t)$ is continuous on $[0, T], v_{k} \neq 0,1,2, \ldots$ and $\psi(t)$ and all the coefficients are continuous $(n+1)$-linear forms on $\mathcal{A}$.

With the above condition, one defines $\mathrm{Pf}_{t=0^{+}} \phi=\psi\left(0^{+}\right)$and the finite part of the integral $\mathrm{Pf}_{t=0^{+}} \int_{0}^{T} \phi(t) d t$ by removing the divergence at $0^{+}$. 
Theorem 2.1 ([CoM], Proposition 4). Let $(\mathcal{H}, D)$ be an unbounded p-summable Fredholm module over $\mathcal{A}$. Suppose for each $\ell \geq 0, C h^{\ell}(t D)$ and $\phi h^{\ell}(t D, D)$ satisfy the condition $(\sharp)$. Then, for any $n>p-1$, n of the same parity as $(\mathcal{H}, D)$, the cocycle in $(b, B)$ bicomplex of $\mathcal{A}$,

$$
\operatorname{Pf}_{c h}^{n}(D):=\sum_{k \geq 0} \operatorname{Pf}_{t=0^{+}} C h^{n-2 k}(t D)
$$

represents the periodic cyclic cohomology Chern character $c_{*}(\mathcal{A}, H, D)$.

\section{Periodic cyclic cohomology Chern character for $X^{\dagger}$}

Let $X^{\dagger}$ be an admissible pseudomanifold with one singular stratum. Now let us apply Theorem 2.1 to compute the periodic cyclic cohomology Chern character for unbounded $p$-summable Fredholm modules over $C_{S A}^{\infty}\left(X^{\dagger}\right)$.

Let $D$ be the signature operator with domain consisting of smooth sections of compact support. As in [Chan], we shall choose a small scaling $\epsilon$ in the conical direction as follows:

$$
\left.g\right|_{c(L) \times N}=\left(\frac{d r^{2}}{\epsilon}+\psi(r)^{2} g^{L}\right) \oplus g^{N}
$$

such that the signature operator $D$ is essentially self-adjoint. We will also denote the self-adjoint extension by $D$.

Even Case: $m=\operatorname{dim} X^{\dagger}$ is even. In [Chan, Section 2.2], for $p>m$, we constructed an even unbounded $p$-summable Fredholm module $(\mathcal{A}, H, D)$, where

$$
\begin{aligned}
& \mathcal{A}=C_{S A}^{\infty}\left(X^{\dagger}\right), \quad \mathcal{H}=\text { signature complex with usual grading, } \\
& D=\bar{d}+\bar{\delta} \quad \text { the signature operator. }
\end{aligned}
$$

Theorem 3.1. Suppose $X^{\dagger}$ is an even-dimensional, admissible Riemannian pseudomanifold with one singular stratum. Under a suitable scaling of the metric in the conical direction, in $(b, B)$ bicomplex of $C_{S A}^{\infty}\left(X^{\dagger}\right)$, for even and non-negative $\alpha$, we have

$$
\begin{aligned}
\operatorname{Pf}_{t=0^{+}} C h^{\alpha}(t D)\left(a^{0}, \ldots, a^{\alpha}\right) & \\
=\frac{1}{\alpha !(2 \pi i)^{\frac{\alpha}{2}}}\left[\int_{M} 2^{\frac{m}{2}} \hat{\mathcal{L}}\left(R\left(g^{M}\right)\right) \wedge a^{0} d a^{1}\right. & \wedge \cdots \wedge d a^{\alpha} \\
& \left.\quad-\eta(L) \int_{N} 2^{\frac{n}{2}} \hat{\mathcal{L}}\left(R\left(g^{N}\right)\right) \wedge a^{0} d a^{1} \cdots \wedge d a^{\alpha}\right],
\end{aligned}
$$

where $\hat{\mathcal{L}}\left(R\left(g^{M}\right)\right)$ and $\hat{\mathcal{L}}\left(R\left(g^{N}\right)\right)$ are the Atiyah-Hirzebruch $\mathcal{L}$-polynomials in the curvature of the Levi-Civita connections of the metrics $g^{M}$ and $g^{N}$ respectively.

Remark on Theorem 3.1. For even $\alpha$ and even $\ell$, the above integral over $N$ vanishes automatically.

Proof. In the following, $a^{i} \in \mathcal{A}, c_{r_{1}, r_{2}}(L)=$ the part of the cone corresponding to $\left(r_{1}, r_{2}\right) \times L$, and $R\left(g^{N}\right)$ is the curvature of Levi-Civita connection of the metric $g^{N}$, etc. 
We will replace the computation on the singular set by one on $c_{0, \infty}(L) \times N$ with the product metric. This is achieved by a singular elliptic estimate [Chan, Proposition 2.9]. Using the results of $[\mathrm{BF}]$, the condition $(\sharp)$ in Theorem 2.1 is satisfied.

Let $\alpha$ be an even and non-negative integer not greater than $m$. Choose $\rho_{0} \in$ $C^{\infty}([0,1])$ with $\rho_{0}(r) \in[0,1]$ such that

$$
\rho_{0}(r)= \begin{cases}0, & r \in\left[0, \frac{3}{4}\right] \\ 1, & r \in\left[\frac{7}{8}, 1\right] .\end{cases}
$$

Define $\rho: X \rightarrow \mathbb{R}$ such that

$$
\rho(x)=\left\{\begin{array}{cl}
1, & x \in M, \\
\rho_{0}(r), & x=(r, \mathfrak{s}, y) \in c(L) \times N .
\end{array}\right.
$$

With the help of a partition of unity, we express $\operatorname{Pf}_{t=0^{+}} C h^{\alpha}(t D)\left(a^{0}, \ldots, a^{\alpha}\right)$ as a sum of contributions from a closed manifold, the cylindrical part and the singular set:

$$
\begin{aligned}
& \operatorname{Pf}_{t=0^{+}} C h^{\alpha}(t D)\left(a^{0}, \ldots, a^{\alpha}\right) \\
= & \operatorname{Pf}_{t=0^{+}} \int_{\Delta_{\alpha}} \int_{X} \operatorname{tr}\left(\gamma a^{0} e^{-t_{1} t^{2} D^{2}}\left[t D, a^{1}\right] \cdots e^{-\left(1-t_{\alpha}\right) t^{2} D^{2}}\right)(x, x) d t_{1} \cdots d t_{\alpha} \\
= & \operatorname{Pf}_{t=0^{+}} C h^{\alpha}\left(t D_{\left(M \cup\left(C_{\frac{3}{4}, 1}(L) \times N\right)\right) \cup-\left(M \cup\left(C_{\frac{3}{4}, 1}(L) \times N\right)\right)}\right)\left(\rho a^{0}, a^{1}, \ldots, a^{\alpha}\right) \\
& +\operatorname{Pf}_{t=0^{+}} \int_{\Delta_{\alpha}} \int_{C_{\frac{3}{4}, 1}(L) \times N} \operatorname{tr}\left(\gamma(1-\rho) a^{0} e^{-t_{1} t^{2} D^{2}}\left[t D, a^{1}\right] \cdots\right)(x, x) d t_{1} \cdots d t_{\alpha} \\
& +\operatorname{Pf}_{t=0^{+}} \int_{\Delta_{\alpha}} \int_{C_{0, \frac{3}{4}}(L) \times N} \operatorname{tr}\left(\gamma a^{0} e^{-t_{1} t^{2} D^{2}}\left[t D, a^{1}\right] \cdots e^{-\left(1-t_{\alpha}\right) t^{2} D^{2}}\right)(x, x) d t_{1} \cdots d t_{\alpha}
\end{aligned}
$$

where $\rho a^{0} e^{-t_{1} t^{2} D^{2}}\left[t D, a^{1}\right] \cdots\left[t D, a^{\alpha}\right] e^{-\left(1-t_{\alpha}\right) t^{2} D^{2}}$ extends to $-\left(M \cup\left(c_{\frac{3}{4}, 1}(L) \times N\right)\right)$ by zero.

We will employ the asymptotic symbolic calculus of global pseudodifferential operators, $\mathfrak{A} \psi D O[\mathrm{Ge} 1]$, with scaling $t$.

Notice that $\left[t D, a^{i}\right] \in \mathfrak{A} \psi D O$ with $\sigma_{t^{-1}}\left(\left[t D, a^{i}\right]\right)=d a+\mathrm{O}(t)$

Case $1 . \ell$ is odd and $n$ is even. Note that

$$
\begin{aligned}
& \int_{c_{\frac{3}{4}, 1}(L) \times N} 2^{\frac{m}{2}} \hat{\mathcal{L}}\left(R\left(g^{X}\right)\right) \wedge \rho a^{0} d a^{1} \wedge \cdots \wedge d a^{\alpha} \\
= & 2^{\frac{m}{2}}\left(\int_{c_{\frac{3}{4}, 1}(L)} \hat{\mathcal{L}}\left(R\left(g^{c(L)}\right)\right) \rho\right) \int_{N} \hat{\mathcal{L}}\left(R\left(g^{N}\right)\right) a^{0} d a^{1} \cdots d a^{\alpha} \\
= & 0 .
\end{aligned}
$$

First, we will consider the contributions from the cylindrical part and the singular set. With the help of a singular elliptic estimate, it suffices to consider the 
computation on the product manifold $c_{0, \infty}(L) \times N$. Since $a^{i} \in C_{S A}^{\infty}\left(X^{\dagger}\right)$, we have

$$
\begin{aligned}
& \int_{c_{\frac{3}{4}, 1}(L) \times N} \operatorname{tr}_{s}\left((1-\rho) a^{0} e^{-t_{1} t^{2} D^{2}}\left[t D, a^{1}\right] \cdots e^{-\left(1-t_{\alpha}\right) t^{2} D^{2}}\right)(x, x) \\
&= \int_{\left(\frac{3}{4}, 1\right) \times L} \operatorname{tr}_{s}\left(\left(1-\rho_{0}(r)\right) e^{-t D_{\left(\frac{3}{4}, 1\right) \times L}^{2}}\right)(x, x) \int_{N} \operatorname{tr}_{s}\left(a^{0} e^{-t_{1} t^{2} D^{2}}\left[t D, a^{1}\right] \cdots\right. \\
&= \mathrm{O}\left(t^{\infty}\right) \\
& \int_{c_{0, \frac{3}{4}}(L) \times N} \operatorname{tr}_{s}\left(a^{0} e^{-t_{1} t^{2} D^{2}}\left[t D, a^{\alpha}\right] e^{-\left(1-t_{\alpha}\right) t^{2} D^{2}}\right)(x, x)+\mathrm{O}\left(t^{\infty}\right) \\
&=\left.\int_{c_{0, \frac{3}{4}}(L)} \operatorname{tr}_{s}\left(e^{\left.-t D_{c_{0, \infty}(L)}^{2}\right)}\right)(x, x) \int_{N}^{-\left(1-t_{\alpha}\right) t^{2} D^{2}}\right)(x, x) \\
&=-\eta(L) \int_{N}\left(a^{0} e^{-t_{1} t^{2} D^{2}}\left[t D, a^{1}\right] \cdots\right)(x, x)+\mathrm{O}\left(t^{\infty}\right) \\
& \operatorname{tr}_{s}\left(a^{0} e^{-t_{1} t^{2} D^{2}}\left[t D, a^{1}\right] \cdots e^{-\left(1-t_{\alpha}\right) t^{2} D^{2}}\right)(x, x)+\mathrm{O}\left(t^{\infty}\right) .
\end{aligned}
$$

So it suffices to show that

$$
\mathrm{Pf}_{t=0^{+}} C h^{\alpha}\left(D_{N}\right)\left(a^{0}, \ldots, a^{\alpha}\right)=\frac{1}{(2 \pi i)^{\frac{\alpha}{2}} \alpha !} \int_{N} 2^{\frac{n}{2}} \hat{\mathcal{L}}\left(R\left(g^{N}\right)\right) a^{0} d a^{1} \cdots d a^{\alpha} .
$$

In order to pass to the signature operator, we will consider the Dirac operator on an even-dimensional smooth closed spin manifold $N$ twisted by a bundle $E$ with curvature $F$. By using Getzler's calculus and the fact $[\mathrm{BF}]$ that the leading symbol of $\left[t D, a^{i}\right]$ and $e^{-t_{i} t^{2} D^{2}}$ commute, we have

$$
\begin{aligned}
& \int_{N} \operatorname{tr}_{s}\left(a^{0} e^{-t_{1} t^{2} D^{2}}\left[t D, a^{1}\right] \cdots\left[t D, a^{\alpha}\right] e^{-\left(1-t_{\alpha}\right) t^{2} D^{2}}\right)(x, x)+\mathrm{O}(t) \\
= & \frac{1}{(2 \pi)^{n}}\left(\frac{2}{i}\right)^{\frac{n}{2}} \int_{N} a^{0} d a^{1} \cdots d a^{\alpha} \operatorname{tr} e^{-F} \operatorname{tr} e^{-\left(|\xi|^{2}-\frac{1}{16} R\left(g^{N}\right) \wedge R\left(g^{N}\right)\right)}+\mathrm{O}(t) \\
= & \frac{1}{\pi^{\frac{n}{2}}(2 \pi i)^{\frac{n}{2}}} \int_{N} a^{0} d a^{1} \cdots d a^{\alpha} \operatorname{ch}(2 \pi i F) \pi^{\frac{n}{2}} \operatorname{det}^{\frac{1}{2}}\left(\frac{R\left(g^{N}\right) / 2}{\sinh \left(R\left(g^{N}\right) / 2\right)}\right)+\mathrm{O}(t) \\
= & \frac{1}{(2 \pi i)^{\frac{n}{2}}} \int_{N} a^{0} d a^{1} \cdots d a^{\alpha} \operatorname{ch}(2 \pi i F) \hat{A}\left(2 \pi i R\left(g^{N}\right)\right)+\mathrm{O}(t) \\
= & \frac{1}{(2 \pi i)^{\frac{n}{2}}}(2 \pi i)^{\frac{n-\alpha}{2}} \int_{N} a^{0} d a^{1} \cdots d a^{\alpha} \operatorname{ch}(F) \hat{A}\left(R\left(g^{N}\right)\right)+\mathrm{O}(t) \\
= & \frac{1}{(2 \pi i)^{\frac{\alpha}{2}}} \int_{N} a^{0} d a^{1} \cdots d a^{\alpha} \operatorname{ch}(F) \hat{A}\left(R\left(g^{N}\right)\right)+\mathrm{O}(t) .
\end{aligned}
$$

Case 2. $\ell$ is even and $n$ is odd. On $c_{0, \infty}(L) \times N$, we have $D=D_{0, \infty} \otimes I+\phi \otimes D_{N}$ (see $[\mathrm{MW}]$ ), where $\phi=(-1)^{k}$ on $L^{2}\left(\Omega^{k}\left(c_{0, \infty}(L)\right)\right)$. Also,

$$
\gamma=\frac{1}{i} \gamma_{1} \gamma_{2}, \quad \gamma_{1}=i^{\frac{\ell+2}{2}} c\left(\operatorname{vol}_{c_{0, \infty}(L)}\right) \otimes I, \quad \gamma_{2}=i^{\frac{n+1}{2}} \phi \otimes c\left(\operatorname{vol}_{N}\right)
$$

where $c\left(v o l_{c_{0, \infty}(L)}\right)$ and $c\left(v o l_{N}\right)$ are Clifford multiplication by volume elements in $c_{0, \infty}(L)$ and $N$ respectively. 
Now $D^{2}=D_{c_{0, \infty}(L)}^{2} \otimes I+I+\otimes D_{N}^{2}$, and

$$
\gamma_{2} D^{2}=D^{2} \gamma_{2}, \quad \gamma_{1} \gamma_{2}=-\gamma_{2} \gamma_{1}, \quad \gamma_{2}\left[D, a^{i}\right]=-\left[D, a^{i}\right] \gamma_{2} .
$$

Therefore, on $c_{0, \infty}(L) \times N$,

$$
\begin{aligned}
& \operatorname{tr}_{s}\left((1-\rho) a^{0} e^{-t_{1} t^{2} D^{2}}\left[t D, a^{1}\right] \cdots\left[t D, a^{\alpha}\right] e^{-\left(1-t_{\alpha}\right) t^{2} D^{2}}\right)(x, x) \\
= & \frac{(-1)^{\alpha}}{i} \operatorname{tr}\left(\gamma_{1}(1-\rho) a^{0} e^{-t_{1} t^{2} D^{2}}\left[t D, a^{1}\right] \cdots\left[t D, a^{\alpha}\right] e^{-\left(1-t_{\alpha}\right) t^{2} D^{2}} \gamma_{2}\right)(x, x) \\
= & \frac{1}{i} \operatorname{tr}\left(\gamma_{2} \gamma_{1}(1-\rho) a^{0} e^{-t_{1} t^{2} D^{2}}\left[t D, a^{1}\right] \cdots\left[t D, a^{\alpha}\right] e^{-\left(1-t_{\alpha}\right) t^{2} D^{2}}\right)(x, x) .
\end{aligned}
$$

Similarly, $\operatorname{tr}_{s}\left((1-\rho) a^{0} e^{-t_{1} t^{2} D^{2}}\left[t D, a^{1}\right] \cdots e^{-\left(1-t_{\alpha}\right) t^{2} D^{2}}\right)(x, x)=0$ on $(0,1) \times L \times N$. Therefore, our results follow from Getzler's calculus as in the previous case.

Corollary 3.2 ([Chan], Theorem 4.1). Suppose $X^{\dagger}$ is an even-dimensional, admissible Riemannian pseudomanifold with one singular stratum. Let $(E, \nabla)$ be a Hermitian vector bundle on $X^{\dagger}$ with a Hermitian connection such that its restriction to the subset $c(L) \times N$ is pulled back from $N$ and $D_{E}$ be the associated twisted signature operator. Under a suitable scaling of the metric in the conical direction, we have

$$
\operatorname{Ind}\left(D_{E}\right)=2^{\frac{m}{2}} \int_{M} \hat{\mathcal{L}}\left(R\left(g^{M}\right)\right) \wedge \operatorname{ch}(E)-\eta(L) 2^{\frac{n}{2}} \int_{N} \hat{\mathcal{L}}\left(R\left(g^{N}\right)\right) \wedge \operatorname{ch}(E)
$$

where $\operatorname{ch}(E)=\operatorname{tr}\left(e^{\frac{i R^{E}}{2 \pi}}\right)$ and $R^{E}$ is the curvature of $(E, \nabla)$.

Proof. The factors $\frac{1}{\alpha !(2 \pi i)^{\frac{\alpha}{2}}}$ compensate for the corresponding factors in

$$
\operatorname{ch}(e)=\operatorname{tr}_{1}(e)+\sum_{k \geq 1}(-1)^{k} \frac{2 k !}{k !}\left(\operatorname{tr}_{2 k+1}\left(e^{\otimes 2 k+1}\right)-\frac{1}{2} \operatorname{tr}_{2 k+1}\left(1 \otimes e^{\otimes 2 k}\right)\right)
$$

to give the precise index formula.

Odd Case: $m=\operatorname{dim} X^{\dagger}$ is odd. Because of the singularity in the asymptotic expansion of the trace of the heat kernel on the cone, we will only consider admissible spaces with conical singularity. That is, $N$ is a point.

By a similar argument, for $p>m$, one can get an odd unbounded $p$-summable Fredholm module $(\mathcal{A}, H, D)$ where

$$
\mathcal{A}=C_{S A}^{\infty}\left(X^{\dagger}\right), \quad \mathcal{H}=L^{2}\left(\wedge^{*} T X\right) \quad \text { and } \quad D=\text { the signature operator. }
$$

Theorem 3.3. Suppose $X^{\dagger}$ is an odd-dimensional, admissible space with conical singularity. Under a suitable scaling of the metric in the conical direction, in $(b, B)$ bicomplex of $C_{S A}^{\infty}\left(X^{\dagger}\right)$, for odd and positive $\alpha$, we have

$$
\mathrm{Pf}_{t=0^{+}} C h^{\alpha}(t D)\left(a^{0}, \ldots, a^{\alpha}\right)=\frac{1}{\alpha !(2 \pi i)^{\frac{\alpha}{2}}} \int_{M} 2^{\frac{m-1}{2}} \hat{\mathcal{L}}\left(R\left(g^{M}\right)\right) \wedge a^{0} d a^{1} \wedge \cdots \wedge d a^{\alpha}
$$

where $\hat{\mathcal{L}}\left(R\left(g^{M}\right)\right)$ and $\hat{\mathcal{L}}\left(R\left(g^{N}\right)\right)$ are the Atiyah-Hirzebruch $\mathcal{L}$-polynomials in the curvature of the Levi-Civita connections of the metrics $g^{M}$ and $g^{N}$ respectively. 
Proof. Note that $d a^{\alpha}=0$ on $c(L)$.

Following the proof of Theorem 3.1, it suffices to consider the twisted Dirac operator on an odd-dimensional smooth closed spin manifold $N$ and show that, for odd and positive $\alpha$,

$$
\begin{aligned}
& \int_{N} \operatorname{tr}\left(a^{0} e^{-t_{1} t^{2} D_{N, E}^{2}}\left[t D_{N, E}, a^{1}\right] \cdots\left[t D_{N, E}, a^{\alpha}\right] e^{-\left(1-t_{\alpha}\right) t^{2} D_{N, E}^{2}}\right)(x, x) \\
= & \frac{1}{(2 \pi i)^{\frac{\alpha}{2}} \sqrt{2 i}} \int_{N} a^{0} d a^{1} \cdots d a^{\alpha} \operatorname{ch}(F) \hat{A}\left(R\left(g^{N}\right)\right)+\mathrm{O}(t) .
\end{aligned}
$$

Note that for an odd operator $P$, we have

$$
\operatorname{Tr}\left(P_{t}\right)=\frac{1}{(2 \pi)^{n}} \frac{2^{\frac{n-1}{2}}}{i^{\frac{n+1}{2}}} \int_{T^{*} N}\left[\left(\sigma\left(P_{t}\right)_{t^{-1}}\right)\right]_{\text {top }} d \xi .
$$

One can now apply Getzler's calculus as before.

Corollary 3.4. Suppose $X^{\dagger}$ is an odd-dimensional, admissible space with conical singularity. Let $D$ be the signature operator and $g \in U_{r}\left(C_{S A}^{\infty}\left(X^{\dagger}\right)\right)$. Under a suitable scaling of the metric in the conical direction, the spectral flow

$$
s f\left(D, g^{-1} D g\right)=-\int_{M} 2^{\frac{m-1}{2}} \hat{\mathcal{L}}\left(R\left(g^{M}\right)\right) c h^{*}(g)
$$

where

$$
c h^{*}(g)=-\sum_{j \geq 0} \frac{j !}{(2 j+1) !} \operatorname{tr}\left(\frac{\left(g^{-1} d g\right)^{2 j+1}}{(2 \pi i)^{j+1}}\right) .
$$

Proof. Note that

$$
s f\left(D, g^{-1} D g\right)=\langle\varphi, g\rangle=\frac{1}{\sqrt{2 \pi i}} \sum_{j \geq 0}(-1)^{j} j ! \varphi_{2 j+1}\left(g^{-1}, g, \ldots, g^{-1}, g\right) .
$$

Then the result follows from Theorem 3.3 (cf. [Ge2]).

\section{ACKNOWLEDGMENT}

This is part of my Ph.D. thesis at The Ohio State University. The author wants to thank Professor H. Moscovici for his encouragement and the referee for his helpful suggestions.

\section{REFERENCES}

$[\mathrm{BF}]$ J. Block and J. Fox, Asymptotic Pseudodifferential Operators and Index Theory, in Comtemp. Math. Vol. 105 (1990), 1-32. MR 91e:58186

[Chan] S. W. Chan, $\mathcal{L}$-classes on Pseudomanifolds with One Singular Stratum, Proc. Amer. Math. Soc. 125 (1997), 1955-1968. CMP 95:11

[Co] A. Connes, Noncommutative Geometry, Academic Press (1994). MR 95j:46063

$[\mathrm{CoM}]$ A. Connes and H. Moscovici, Transgression and the Chern Character of FiniteDimensional K-cycles, Commun. Math. Phys. 155 (1993), 103-122. MR 95a:46091

[Ge1] E. Getzler, Pseudodifferential Operators on Supermanifolds and the Atiyah-Singer Index Theorem, Commun. Math. Phys. 92 (1983), 163-178. MR 86a:58104

[Ge2] E. Getzler, The Odd Chern Character in Cyclic Homology and Spectral Flow, Topology 32 No.3 (1993), 487-507. MR 95c:46118

[MW] H. Moscovici and F.-B. Wu, Pontryagin Forms for Manifolds with Framed Singular Strata, Geom. and Func. Analysis 5 No.4 (1995), 702-728. MR 96g:58185

Department of Mathematics, The Ohio State University, Columbus, Ohio 43210

E-mail address: swchan@math.ohio-state.edu 